\title{
PRODUCTION OF POLY(3-HYDROXYBUTYRATE-CO-3- HYDROXYVALERATE) BY MARINE ARCHAEA Haloferax mediterranei DSM 1411 WITH YEAST EXTRACT AS NUTRIENT SOURCE
}

\author{
Susiana Melanie ${ }^{1 *}$ and James Winterburn ${ }^{2}$ \\ ${ }^{1}$ Research and Development Center for Marine and Fisheries Product Processing and Biotechnology \\ JI. KS. Tubun Petamburan VI, Jakarta 10260 Indonesia \\ ${ }^{2}$ School of Chemical Engineering and Analytical Science, The University of Manchester, M13 9PL United Kingdom
}

Article history:

Received: 10 August 2017; Revised: 30 September 2017; Accepted: 30 November 2017

\begin{abstract}
In many countries, most of household goods are made from polymer or plastic. The polymer manufacturing industry has been relying on fossil fuel-based raw materials which are non-biodegradable. Plastics derived from these non-biodegradable sources will be difficult to degrade in nature, which in turn will pollute the environment and harm the ecosystem. One way to reduce the ecosystem damages caused by petroleum-based plastic is by using biodegradable materials for plastic industry, such as Polyhydroxyalkanoates (PHA). Poly (3-hydroxybutyrateco-3-hydroxyvalerate) or PHBV is an example of PHA that can be produced by several types of microorganism, one of them is marine archaea Haloferax mediterranei. The objective of this study is to determine the productivity of marine archaea $\mathrm{H}$. mediterranei cultures to produce PHBV with yeast extract as main nutrient sources. Experiments and analysis were conducted in triplicate and batch operating system. During the fermentation process, optical density, glucose levels, phosphorus levels, $\mathrm{pH}$, and dry cell weight (DCW) were observed. The final product was then analysed using GC-MS to determine 3HB (3-hydroxybutyrate) and 3HV (3-hydroxyvalerate) fraction composition in the PHBV. The results showed that the maximum specific growth rate $(\mu)$ and doubling time (Td) of the $H$. mediterranei were 0.1258 hours $^{-1}$ and 5.51 hours, respectively. $H$. mediterranei biomass increased until it reached stationary phase after 95 hours incubation. The PHBV content, dry biomass and yield of PHBV to substrate were $2.62 \mathrm{~g} / \mathrm{L}, 29.1 \%$ and $0.27 \mathrm{~g} / \mathrm{g}$, respectively.
\end{abstract}

Keyword: biopolymer, Poly (3-hydroxybutyrate-co-3-hydroxyvalerate), PHBV, Haloferax mediterranei, yeast extract

\section{Introduction}

Most of the daily life equipments are made of polymer or plastic. The polymer manufacturing industry relies on non-biodegradable petroleum-based raw materials that can pollute the environment, especially marine ecosystems. Plastic waste can endanger the ecosystem with their toxic compounds such as colourings, flame retardants, and plasticiser. Around 10-20 million tonnes of plastics are discarded to the oceans, which then can disintegrate into small pieces. One of which is in the form of micro plastic that can be consumed by sea animals. As a result the plastics chemical compounds are transferred through the food chain (Gourmelon, 2015). These problems are gaining people awareness around the world and rising demand for greener sustainable polymers.

Polyhydroxyalkanoate (PHA), which more environmentally friendly for packaging and more safety for biomedical use, is one of the solutions to reduce the use of non-biodegradable polymer materials, (Anon., 2017; Carvalho, 2013; Chen, 2009; Kaewkannetra, 2012; Pal, Paulson, \& Rousseau, 2009). It was reported that the market for PHA products only accounts about $1 \%$ of the total global plastics market annually (Anon., 2016). This result is possibly due to the PHA characteristics that still not

${ }^{*}$ Corresponding author.

E-mail: susianam@yahoo.com 
perfectly meet the market expectation, especially their elasticity and product homogeneity properties. However, the desired characteristics can be achieved by combining two different monomers of PHA, referred as copolymer. The advantages of the copolymer are its wider application and customisable to meet market needs. Poly (3-hydroxybutyrate-co-3-hydroxyvalerate) or PHBV is an example of a PHA consisting of two homopolymers of poly-3-hydroxybutyrates (3HB) and poly-3-hydroxyvalerates (3HV) (Hermann-Krauss et al., 2013; Koller et al., 2007; Koller, Salerno, Muhr, Reiterer, \& Braunegg, 2012).

One of the marine archaea that can produce PHBV through the fermentation process is Haloferax mediterranei. PHBV is accumulated as an inclusion body in a condition when carbon stocks excess and limited nutrient (such as nitrogen, phosphorus, dissolved oxygen and other micro components) content. The monomer ratio in PHBV will affect the mechanical and physical properties of the biopolymer.

The major challenges and drawback of PHA production are the complication of the biosynthesis process, the high cost of production, and disparity in polymer properties. Up till now, PHBV synthesis has been studied to produce product with specific physiochemical and mechanical properties from various carbon source: whey sugars, rice-based ethanol stillage, dairy residues (Bhattacharyya et al., 2014; Bosco \& Chiampo, 2010; Koller et al., 2007) and nutrient (e.g. nitrogen, phosphorus, dissolved oxygen) limitation. However, their research only focuses on the carbon source and initial nitrogen concentration, but lack of information about the phosphorus concentration during the fermentation. Phosphorus was obtained solely from yeast extract.

The objective of study was to determine the productivity of marine archaea cultures $H$. mediterranei to produce PHBV with yeast extract as main nutrient sources. H. mediterranei will produce PHBV at extreme conditions with excess carbon and limited nutrients.

\section{Material and Methods}

The experiment in this study comprises of medium preparation, inoculum preparation, culture of $\mathrm{H}$. mediterraneiDSM 1411 (DSMZ, Germany), extraction, and product analysis. The analytical procedures were done in triplicate.

\subsection{Medium Preparation}

All the glassware used in the experiment were sterilised in an autoclave $\left(121^{\circ} \mathrm{C}\right.$ for 20 minutes at 15 psig). H. mediterranei DSM 1411 was obtained from DSMZ and maintained in agar plates using base medium which consists of $\mathrm{NaCl} 156 \mathrm{~g} / \mathrm{L}, \mathrm{MgCl}_{2} \cdot 6 \mathrm{H}_{2} \mathrm{O}$ $13 \mathrm{~g} / \mathrm{L}, \mathrm{MgSO}_{4} \cdot 7 \mathrm{H}_{2} \mathrm{O} 20 \mathrm{~g} / \mathrm{L}, \mathrm{CaCl}_{2} \cdot 6 \mathrm{H}_{2} \mathrm{O} 1 \mathrm{~g} / \mathrm{L}, \mathrm{KCl} 4$ $\mathrm{g} / \mathrm{L}, \mathrm{NaHCO}_{3} 0.2 \mathrm{~g} / \mathrm{L}, \mathrm{NaBr} 0.5 \mathrm{~g} / \mathrm{L}$, Yeast extract 5 $\mathrm{g} / \mathrm{L}$, Glucose $10 \mathrm{~g} / \mathrm{L}$, and $15 \mathrm{~g}$ of Agar. Medium for inoculum culture was using the same component but without agar.

\subsection{Inoculum Preparation}

For inoculum, one colony was used to inoculate $10 \mathrm{~mL}$ of liquid medium in a $50 \mathrm{~mL}$ conical tube and incubated at $37^{\circ} \mathrm{C}$. After $24 \mathrm{~h}$, the first inoculum was transferred to a second inoculum of $100 \mathrm{~mL}$ medium in $250 \mathrm{~mL}$ Erlenmeyer flasks, cultivated under the same conditions for $24 \mathrm{~h}$. Shake flasks fermentation were inoculated with $10 \%(\mathrm{v} / \mathrm{v})$ inoculum.

\subsection{Culture of $\boldsymbol{H}$. mediterranei}

The experiment used a standard yeast extract based medium as described previously in medium preparation section. The nitrogen and phosphorus source were provided by yeast as a complex nutrient source. The culture was conducted in triplicates. Shake flasks fermentation ( 1 L erlenmeyer flasks) were inoculated with $10 \%(\mathrm{v} / \mathrm{v})$ inoculum, incubated at $37^{\circ} \mathrm{C}$ and $200 \mathrm{rpm}$ for a week and observed. Samples were taken every 6 hours to measure optical density, $\mathrm{pH}$, phosphorus concentration and glucose concentration (Guell, 2015; Rodriguez-Valera, RuizBerraquero, \& Ramos-Cormenzana, 1980; Wang, Liu, $\& Q i, 2014)$.

\subsection{PHA Extraction}

H. mediterranei grows in high salinity in nature, so it was easy to extract the PHBV product from the cells. The cells will lyse if contacted with distilled water. The biomass was rinsed with distilled water for cell lysis and centrifuged (MiniSpin, Eppendorf) at $13000 \mathrm{rpm}(11337 \times \mathrm{g})$ for 5 minutes. The PHBV pellet was then dried at $60^{\circ} \mathrm{C}$ until constant weight (Bhattacharyya et al., 2012; Chen, Wang, Wei, Liang, \& Qi, 2011; Don, Chen, \& Chan, 2006; Han, Wu, Hou, Zhao, \& Xiang, 2015).

\subsection{Product Analysis}

\subsubsection{Optical density}

Optical density is used to measure the cell growth, using the UV-visible spectrophotometer (UVmini-1240, Shimadzu). $1 \mathrm{~mL}$ of culture broth was centrifuged (MiniSpin, Eppendorf) at $13000 \mathrm{rpm}(11337 \times \mathrm{g})$ for 5 min to separate the supernatant from the pellet. The pellet then was resuspended with $1 \mathrm{~mL}$ of washing 
solution $(10 \% \mathrm{w} / \mathrm{v}$ of $\mathrm{NaCl})$. Optical density was measured with a blank of washing solution as a comparison at $\lambda=600 \mathrm{~nm}$ (Chen, Don, \& Yen, 2006; Rodriguez-Valera et al., 1980; Wang et al., 2006).

\subsubsection{Dry cell weight (DCW)}

Dry cell weight is used to determine the growth rate of $H$. mediterranei culture. Dry cell weight was measured gravimetrically with 24 hours interval in triplicate. The dry weight of the cells pellet was obtained by spinning $3 \mathrm{~mL}$ of culture broth at 13.000 rpm $(11.337 \times \mathrm{g})$ for $5 \mathrm{~min}$, and the pellet was dried at $60{ }^{\circ} \mathrm{C}$ until reach constant weight (Bhattacharyya et al., 2012).

\subsection{3. pH measurement}

The $\mathrm{pH}$ of the culture broth was measured every 24 hour. $H$. mediterranei, like most of the halophile archaea, grows on optimal pH of 7.0 (Bowers \& Wiegel, 2011).

\subsubsection{Glucose and phosphorus measurement}

Glucose concentration of the culture was measured with TRUEresult (Nipro Diagnostic, USA), while phosphorus concentration was measured with Vanadate-Molybdate Method (AOAC, 1995). The absorbance of the samples was measured at $470 \mathrm{~nm}$ using UV-visible spectrophotometer (UVmini-1240, Shimadzu). These measurements were conducted for every 24 hour.

\subsubsection{PHBV content and $3 H \mathrm{~V}$ molar fraction}

The PHBV content and $3 \mathrm{HV}$ molar fraction in dry biomass were analysed using gas chromatography mass spectrometry (GC-MS) (Agilent Technologies,
USA). The dried samples need to be prepared before analysis with GC-MS equipment as described by Chen et al. (2011). Briefly, the dried samples were added with mixture reagent consist of $1 \mathrm{~mL}$ chloroform, $0.85 \mathrm{~mL}$ methanol, and $0.15 \mathrm{~mL}$ sulphuric acid.

\section{Results and Discussions}

This experiment used a yeast extract based medium as the main nutrient source and glucose as carbon source for the culture of $H$. mediterranei. After $48 \mathrm{~h}$ of incubation, the culture medium colour changed from clear yellowish to white pink (Figure 1a). This pink growth appearance was also captured by previous research conducted by Rodriguez-Valera et al. (1980), where the pink growth faintly appeared during the second or third day after incubation. The growth of the cells after $72 \mathrm{~h}$ (day 3 ) was clearly seen by the culture colour, since it became dense as shown in Figure 1b.

Optical density was measured to describe the biomass formation in the culture medium. The optical density of the samples can be seen in Figure 2. The diagram shows that the culture growth has a short lag phase, only for the first 6 hours of incubation. The short lag phase is likely because the medium used in this experiment is the same as that in the inoculum medium, and consequently the adaptation of the $H$. mediterranei cells to the new medium was accomplished fairly rapidly.

After the lag phase, the growth of $H$. mediterranei started to enter the exponential growth phase until $t$ $=71$ hours, where the absorbance at OD600 nm reading was 20.3. After 71 hours, the growth rate started to slow down until $t=95$ hours where the maximum OD600 $\mathrm{nm}$ reading was 23.8 . The optimum

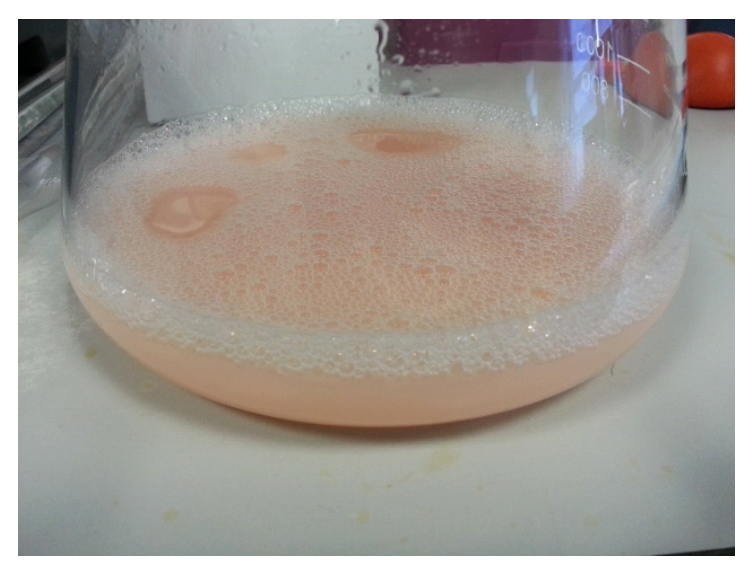

(a)

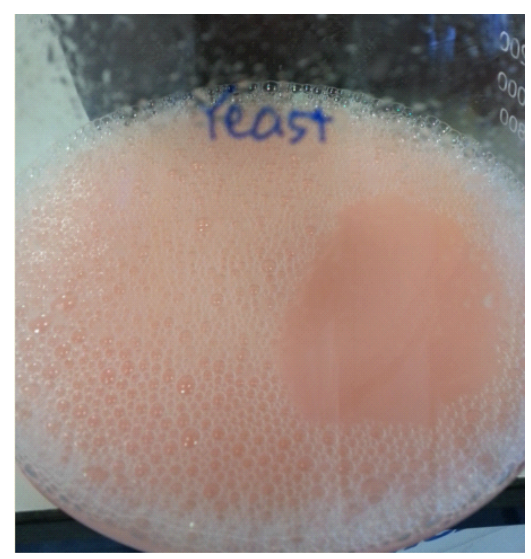

(b)

Figure 1. (a) Culture of $H$. mediterranei in yeast extract medium after $48 \mathrm{~h}$ and (b) after $72 \mathrm{~h}$ in $1 \mathrm{~L}$ erlenmeyer flasks. 
growth rate was reached at interval 6 to 72 hours. After 95 hours, the growth of $H$. mediterranei started to enter the stationary phase.

Figure 2 shows the relationship between optical density (OD600 nm) and dry cell weight (DCW) of $H$. mediterranei fermentation. After a short lag phase for the first 6 hours, the amount of biomass was increased proportionally to the optical density, until it reached the stationary phase after 95 hours. However, there was a disparity between the dry cell weight and the optical density. The dry cell weight was still increasing while the optical density started to enter the stationary phase, even though the increase of the dry cell weight slowed down after 95 hours. A likely explanation of this condition is that the cells were still accumulating PHA inside themselves. The cells did not multiply in amount but grew in size. This phenomenon is consistent with the previous research conducted by Lillo and Rodriguez-Valera (1990), where the cells still accumulate PHA after the stationary phase and decrease at certain time after that. Another reasonable reason for this is the presence of residual biomass due to the cell lysis (Grousseau et al., 2014).

The optical density of $H$. mediterranei culture was used to calculate the specific growth rate $(\mu)$. The graphical determination of the growth rate can be plotted in a diagram as shown in Figure 3. When $\mathrm{Ln}(\mathrm{X})$ $X 0)$ is plotted against time, the slope of linear regression is equal to the specific growth rate. The maximum specific growth rate was obtained between 0 to 24 hours after inoculation. The maximum specific growth rate and doubling time of $\mathrm{H}$. mediterranei fermentation was $0.1258 \mathrm{~h}^{-1}$ and $5.51 \mathrm{~h}$ with the value of $R^{2}=0.9976$ for the linear regression. The doubling time is a valuable parameter to estimate the doubling ability of the cells. This parameter is very useful for a scaled-up process.

The supernatant from the first centrifugation was collected for $\mathrm{pH}$ measurement, where the $\mathrm{pH}$ was measured every 6 hours. The results of $\mathrm{pH}$ measurement can be seen in Figure 4. The culture medium started at an adjusted $\mathrm{pH} 7.0$, but after 24 hours the $\mathrm{pH}$ of fermentation process of $\mathrm{H}$. mediterranei dropped to 6.1 and increased again after 95 hours. The $\mathrm{pH}$ finally reached $\mathrm{pH} 7.1$ at the end of the fermentation process. This is similar with the phenomenon captured by Rodriguez-Valera et al. (1980) where a non-buffered liquid medium with glucose showed that the $\mathrm{pH}$ decreased from 7 to 5.8 after $24 \mathrm{~h}$ incubation. A possible explanation of this phenomenon is that the fermentation becomes slightly acidic, which is related to the metabolism of the cells during the fermentation process. A study by Saharan, Grewal and Kumar (2014) mentioned that mixture of acetic acid and propionic acid can be used as carbon substrate to enhance the culture with an organic load. Grousseau et al. (2014) also have succeeded in running experiments with butyric, propionic, and acetic acid as a substrate on PHBV production by Cupriavidus necator. The decreased $\mathrm{pH}$ could occur when acidic compounds were produced during the fermentation but were consumed again afterwards, rendering the $\mathrm{pH}$ of the culture back to the initial $\mathrm{pH}$.

Figure 5 shows the relationship between the glucose concentration of the culture broth and dry cell

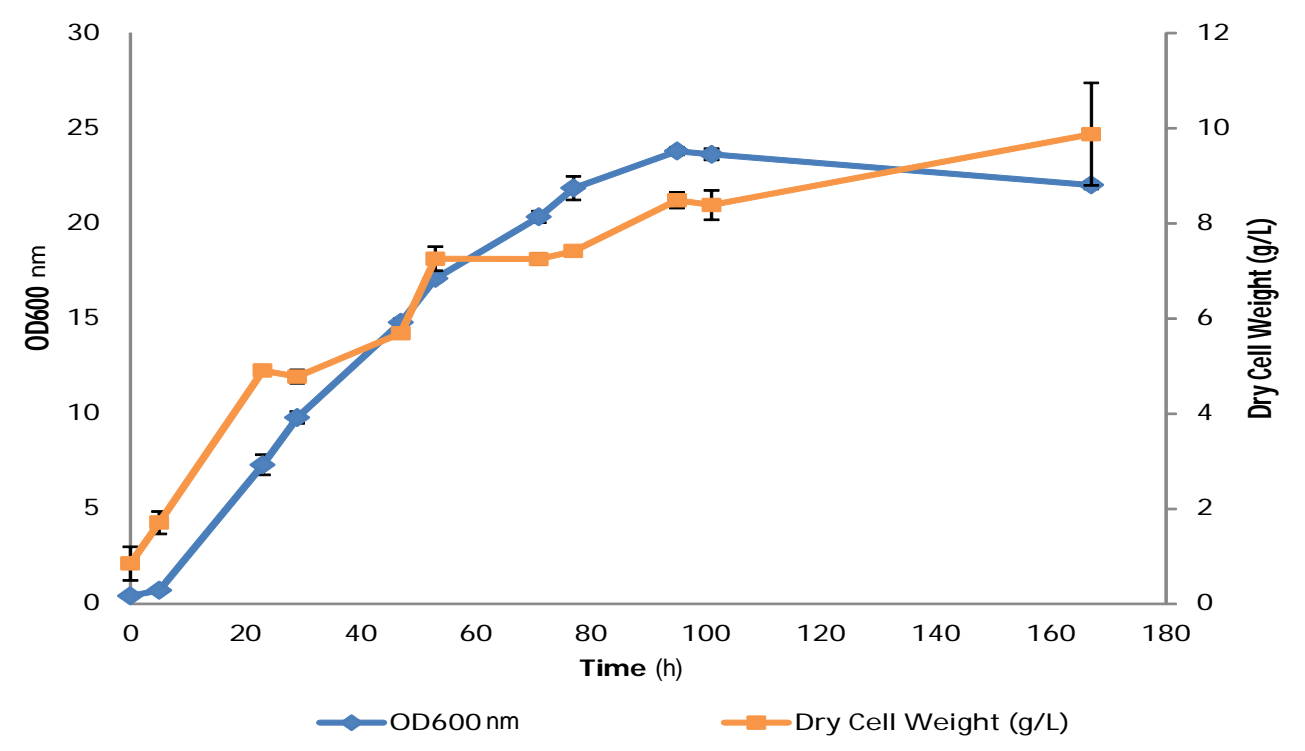

Figure 2. The relationship between $\mathrm{OD} 600 \mathrm{~nm}$ and Dry cell weight (DCW) of $H$. mediterranei fermentation in yeast extract based medium. 


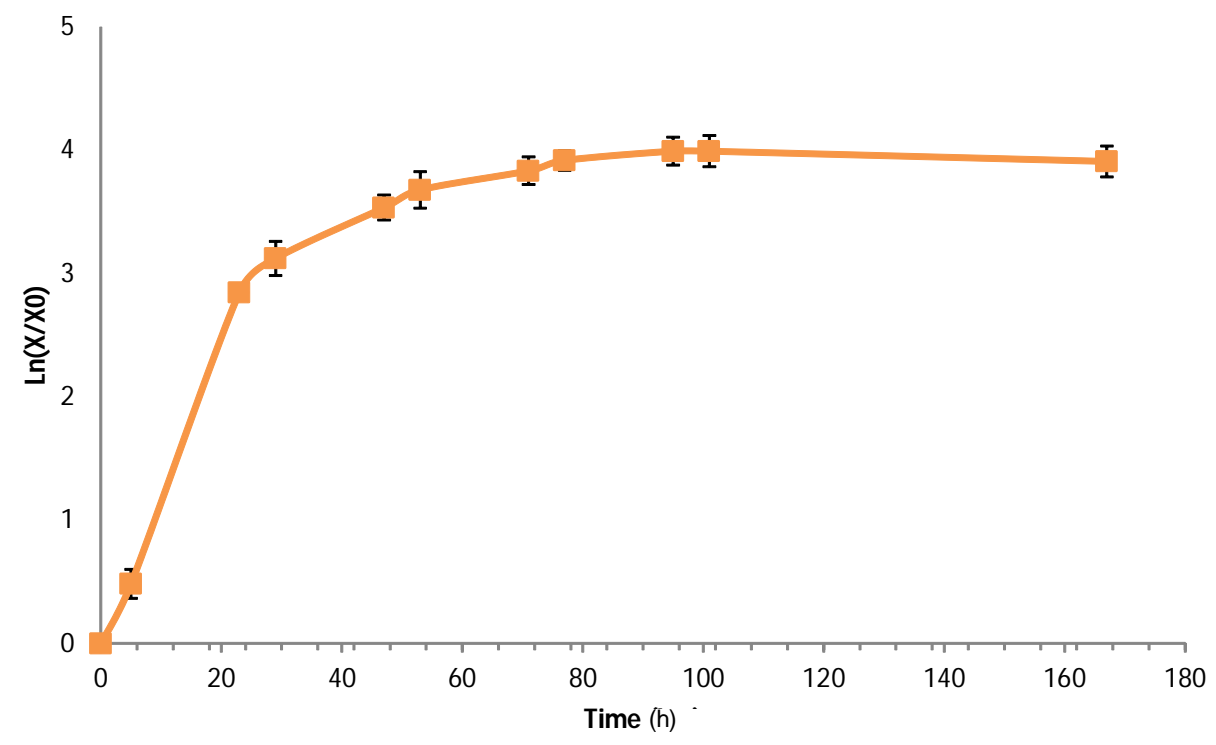

Figure 3. Graphical determination of the $H$. mediterranei specific growth rate.

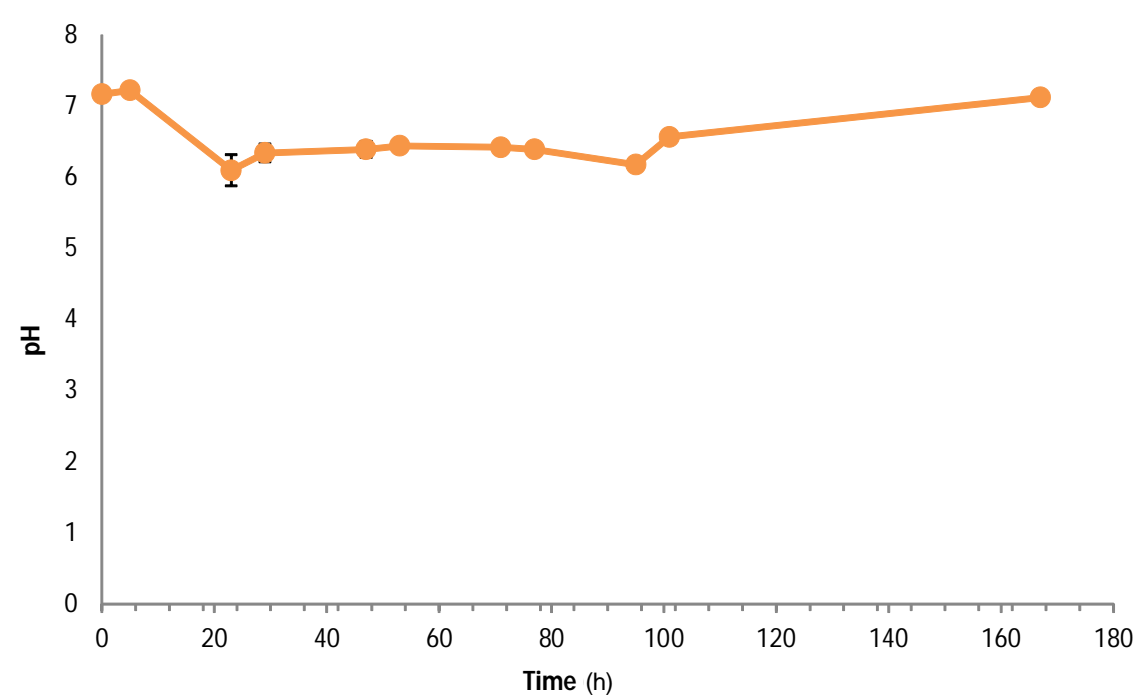

Figure 4. pH value of $H$. mediterranei fermentation in yeast extract based medium.

weight of the biomass. It indicates that the glucose was completely consumed by $H$. mediterranei after 95 hours incubation. It is consistent with the previous research conducted by Chen et al. (2011) where the dry cell weight has been increased with the decrease of glucose concentration. The decreasing of the carbon source were in contrary to the dry weight of the cell as captured in the study of PHBV from $H$. mediterranei by Koller, Chiellini and Braunegg (2015) and PHBV production from Escherichia coliby Wang et al. (2014)

The dry cell weight shows increasing values during the fermentation proportionally to the glucose consumption. The dry cell weight increased while the glucose concentration decreased. In every experiments of PHA production, a similar model always follows as in eubacteria (Koller et al., 2015; Sankhla, Bhati, Singh, \& Mallick, 2010; Wang et al., 2014). However, in this experiment a unique phenomenon occurred after 95 hours incubation. The dry cell weight over time was still increased while the glucose concentration was already completely consumed. This condition may occur because there was still another carbon source other than glucose existed in the medium. Yeast extract are well known as complex nutrient source and also as carbon source. It is likely that the cells were still growing because there was still another carbon source, namely from yeast extract.

Figure 6 it can be seen that the phosphorus concentration was decreased from the beginning of 


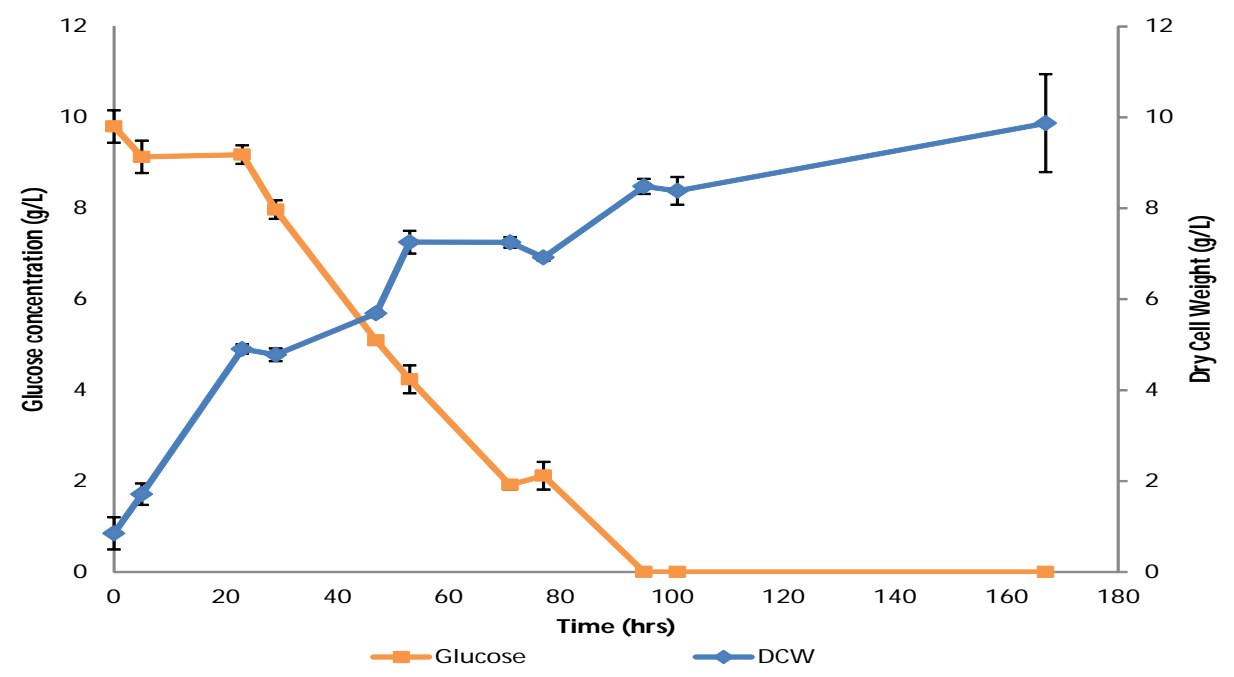

Figure 5. The relationship between glucose concentration of the culture broth and dry cell weight of the biomass

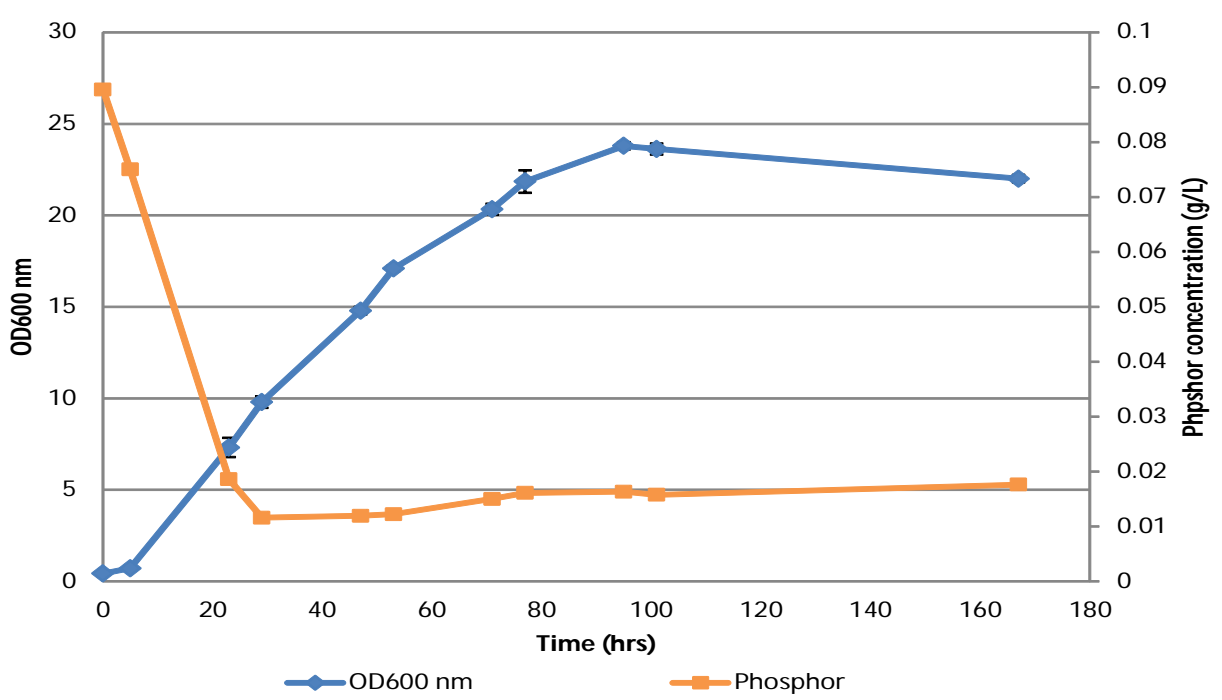

Figure 6. Relationship between optical density and phosphorus concentration

the fermentation process, while the optical density of the fermentation process increased up to the limit of 95 hours. This result confirms the experiment conducted by Grousseau et al. (2014), where lower phosphate concentrations lead to low PHA production, and proportional to the decrease in cell growth. Phosphorus is needed for the growth of $H$. mediterranei during the fermentation process. The phosphorus concentration continued decrease until reached 29 hours after incubation, but began to show constant concentration after 29 hours. The possible explanation for this condition is that the cell of $H$. mediterranei grows with the support of phosphorus salt, but when the phosphorus is limited, it begins to accumulate the PHA.

The dry cell weight of the sample was weighed then further analysed with GC-MS. The advantage of using GC-MS is that the equipment can determine the components in the sample by comparing the retention time of each component in the sample with the library data. The results of the GC-MS analysis consist of retention time, peak area, and the components of the samples. The GC-MS graph result for the final PHA product using yeast extract based medium can be seen in Figure 7. The 3HB (3-hydroxy butanoic acid methyl ester) fraction have the greatest peak area with retention time at 4.775 , while $3 \mathrm{HV}(3-$ hydroxy pentanoic acid methyl ester) fraction came at a retention time of 5.323 .

From the $\mathrm{GC}$ results, the concentration of $3 \mathrm{HB}$ and $3 \mathrm{HV}$ fraction in total dried biomass can be calculated in $\mathrm{g} / \mathrm{L}$. Furthermore, the concentration of the components was converted into percentage and plotted against time (Figure 8). The 3HV fraction 


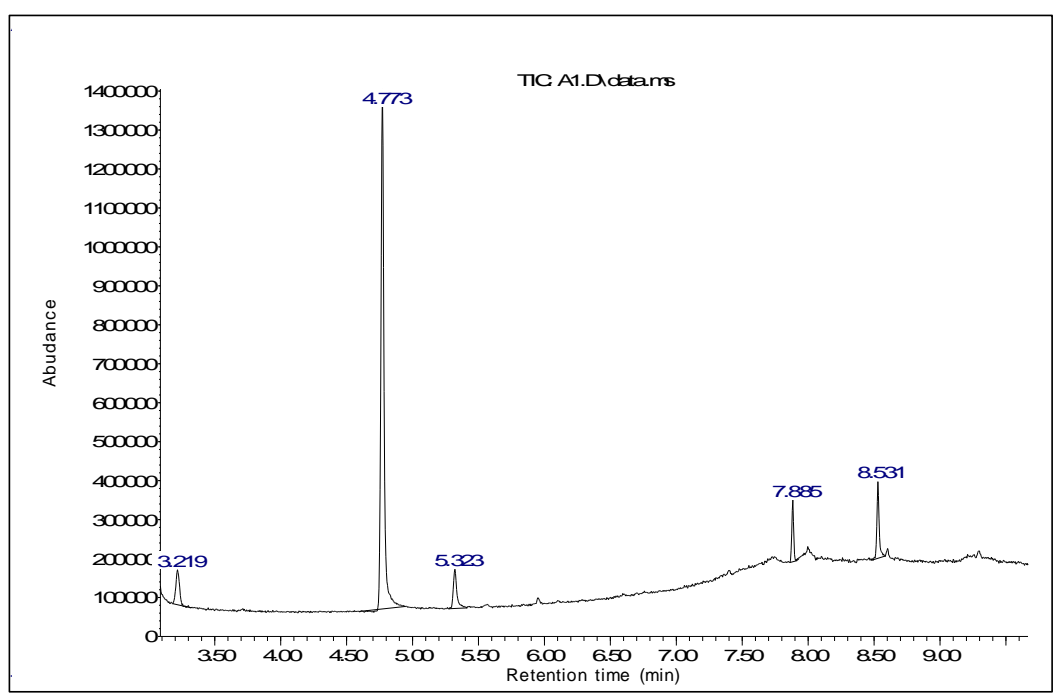

Figure 7. GC-MS curve for final PHA product using yeast extract based medium.

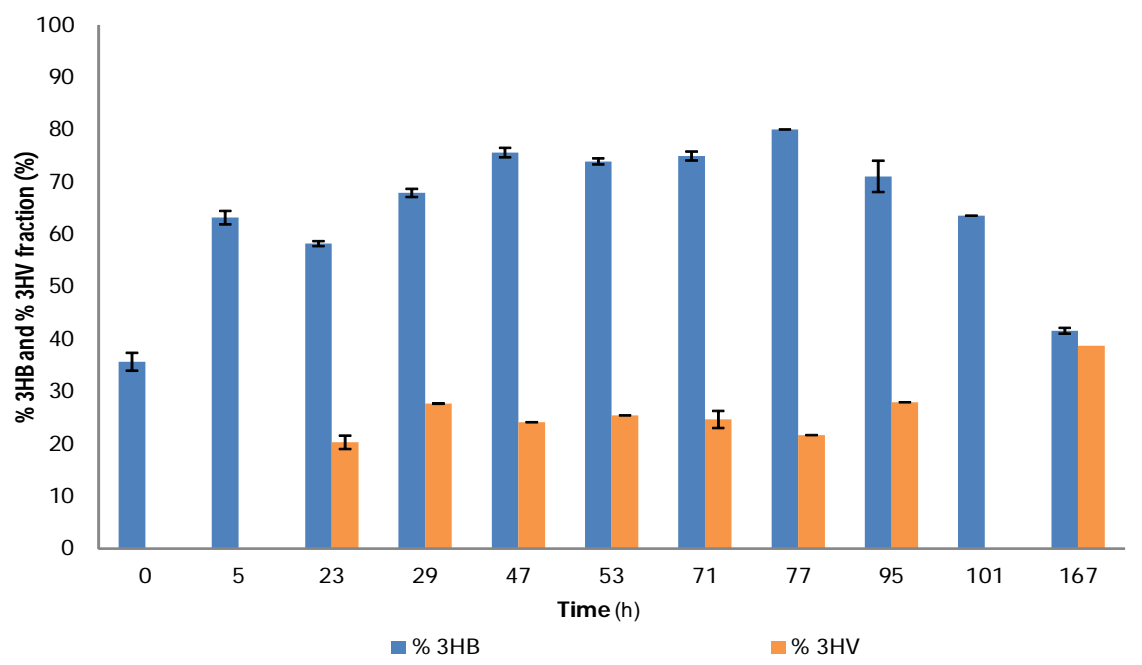

Figure 8. Percentage of $3 \mathrm{HB}$ and $3 \mathrm{HV}$ fraction

appread in the GC result after 23 hours of incubation. It is almost certain that this because the $3 \mathrm{HV}$ fraction was not formed yet at the early stage of $H$. mediterranei growth. The samples from 77 hours and 101 hours after incubation also did not appear in the $\mathrm{GC}$ results. It is likely that limitation of this determination method might cause error, since the sample amount was very small $(10 \mathrm{mg})$.

The $3 \mathrm{HB}$ fraction in the dry biomass was begun with a low amount at the start of incubation, and then increased during the fermentation process until it reached its maximal $6.0 \mathrm{~g} / \mathrm{L}$ at 95 hours after incubation. This increase in $3 \mathrm{HB}$ was in accordance with the dry cell weight. The $3 \mathrm{HB}$ content decreased after 95 hours, which might occur due to the absence of glucose. The PHA in the cells has a function as energy and carbon storage during unfavourable environmental conditions (Rudnik, 2013). Therefore, when there was no carbon source, the $H$. mediterranei could use the carbon storage from PHBV.

The $3 \mathrm{HV}$ fraction in dry biomass increased during the fermentation process, and continued to rise even though the $3 \mathrm{HB}$ concentration decreased. A possible explanation may be because the percentage of $3 \mathrm{HV}$ fraction against $3 \mathrm{HB}$ fraction became higher. This phenomenon can be an advantage because it means that the PHBV can be customised to meet market demand by limiting the duration of fermentation to specific times where the PHBV composition satisfies the requirements. 
Table 1. The growth kinetics parameter and productivity of $H$. mediterranei in a yeast extract based medium

\begin{tabular}{lcccccccccc}
\hline $\begin{array}{l}\text { Nutrient } \\
\text { source }\end{array}$ & $\begin{array}{c}\text { Conc. } \\
(\mathbf{g} / \mathbf{L})\end{array}$ & $\begin{array}{c}\text { Operation } \\
\text { mode }\end{array}$ & $\begin{array}{c}\mu \\
\left(\mathbf{h}^{-1}\right)\end{array}$ & $\begin{array}{c}\mathbf{T d} \\
\mathbf{( h )}\end{array}$ & $\begin{array}{c}\Delta \mathbf{X} \\
(\mathbf{g} / \mathbf{L})\end{array}$ & $\begin{array}{c}\Delta \mathbf{S} \\
(\mathbf{g} / \mathbf{L})\end{array}$ & $\begin{array}{c}\text { PHBV } \\
(\mathbf{g} / \mathbf{L})\end{array}$ & $\begin{array}{l}\mathbf{Y}_{\mathrm{X} / \mathbf{s}} \\
\mathbf{Y}_{\text {PHBV/S }}\end{array}$ & $\mathbf{Y}_{\text {PHBV/X }}$ \\
\hline Yeast extract & 5 & Batch & 0.13 & 5.5 & 9 & 9.8 & 2.62 & 0.92 & 0.27 & 0.291 \\
\hline
\end{tabular}

Note:

$\mu$ : specific growth rate

Td : doubling time

$\Delta \mathrm{X}$ : total biomass

$\Delta S$ : total substrate

$Y_{\text {XJS }}$ : yield biomass respect to substrate

$Y_{\text {PHBV/S }}$ : yield PHBV respect to substrate

$Y_{\text {PHBVX: }}$ yield PHBV respect to biomass

In this experiment, $H$. mediterranei culture produced an accumulated $2.62 \mathrm{~g} / \mathrm{L} \mathrm{PHBV}$ with $29.1 \%$ of dry biomass and yield $Y_{\text {PHBV } / S}$ of $0.27 \mathrm{~g} / \mathrm{g}$ (Table 1 ), which is comparable to the results of study conducted by Koller et al. (2015). With the same carbon source Koller et al. (2015) reported $0.21 \mathrm{~g} / \mathrm{L}$ PHBV produced with $Y_{\text {PHBV/S }}$ of approximately $0.23 \mathrm{~g} /$ g.

\section{Conclusions}

In the stationary phase, $H$. mediterraneicells did not increase in number of cells. Glucose as a carbon source was used for cell growth but when the glucose condition is depleted, the cell begins to consume the PHBV that forms as a carbon source. Phosphorus is necessary in the process of growth of $H$. mediterranei cells and is a limiting nutrient in the PHBV formation process. PHBV was accumulated in cells when phosphorus concentrations were limited. The composition of the $3 \mathrm{HB}$ and $3 \mathrm{HB}$ fractions in the PHBV polymer can be controlled by set the duration of fermentation time.

H. mediterranei culture in this experiment showed a promising productivity with specific growth rate of $0.13 \mathrm{~h}^{-1}$ and a doubling time of $5.5 \mathrm{~h}$. The accumulation of PHBV was $2.62 \mathrm{~g} / \mathrm{L}$ with $29.1 \%$ of dry biomass and yield $Y_{P H B V / S}$ of $0.27 \mathrm{~g} / \mathrm{g}$.

\section{References}

Anonim. (2016). Bioplastics Market Data. Retrieved April 24, 2016, from http://www.european-bioplastics.org/ market/

Anonim. (2017). Global Biodegradable Plastics Market 2016-2022. Retrieved July 25, 2017, from https:// globenewswire.com/news-release/2017/04/17/ 961228/0/en/Global-Biodegradable-Plastics-Market2016-2022.html
AOAC. (1995). Official methods of analysis of AOAC International. In Arlington, Va.: AOAC Intl. pv (16th ed.). Arlington, USA.

Bhattacharyya, A., Pramanik, A., Maji, S. K., Haldar, S., Mukhopadhyay, U. K., \& Mukherjee, J. (2012). Utilization of vinasse for production of poly-3(hydroxybutyrate-co-hydroxyvalerate) by Haloferax mediterranei. AMB Express, 2(1), 34. https://doi.org/ 10.1186/2191-0855-2-34

Bhattacharyya, A., Saha, J., Haldar, S., Bhowmic, A., Mukhopadhyay, U. K., \& Mukherjee, J. (2014). Production of poly-3-(hydroxybutyrate-cohydroxyvalerate) by Haloferax mediterranei using rice-based ethanol stillage with simultaneous recovery and re-use of medium salts. Extremophiles, 18(2), 463-470. https://doi.org/10.1007/s00792-0130622-9

Bosco, F., \& Chiampo, F. (2010). Production of polyhydroxyalcanoates (PHAs) using milk whey and dairy wastewater activated sludge. Production of bioplastics using dairy residues. Journal of Bioscience and Bioengineering, 109(4), 418-421. https://doi.org/10.1016/j.jbiosc.2009.10.012

Bowers, K. J., \& Wiegel, J. (2011). Temperature and pH optima of extremely halophilic archaea: A mini-review. Extremophiles, 15(2), 119-128. https://doi.org/ 10.1007/s00792-010-0347-y

Carvalho, A. J. F. (2013). Starch: major sources, properties and applications as thermoplastic materials. In S. Ebnesajjad (Ed.), Handbook of Biopolymers and Biodegradable Plastics Properties, Processing, and Applications (pp. 129-152). Elsevier. https://doi.org/10.1016/B978-1-4557-2834-3.000070

Chen, C. W., Don, T. M., \& Yen, H. F. (2006). Enzymatic extruded starch as a carbon source for the production of poly(3-hydroxybutyrate-co-3-hydroxyvalerate) by Haloferax mediterranei. Process Biochemistry, 41(11), 2289-2296. https://doi.org/10.1016/ j.procbio.2006.05.026

Chen, G.-Q. (2009). A microbial polyhydroxyalkanoates (PHA) based bio- and materials industry. Chemical 
Society Reviews, 38(8), 2434-2446. https://doi.org/ $10.1039 /$ b812677c

Chen, Q., Wang, Q., Wei, G., Liang, Q., \& Qi, Q. (2011). Production in Escherichia coli of poly(3hydroxybutyrate-co-3-hydroxyvalerate) with differing monomer compositions from unrelated carbon sources. Applied and Environmental Microbiology, 77(14), 4886-4893. https://doi.org/10.1128/ AEM.00091-11

Don, T.-M., Chen, C. W., \& Chan, T.-H. (2006). Preparation and characterization of poly(hydroxyalkanoate) from the fermentation of Haloferax mediterranei. Journal of Biomaterials Science, Polymer Edition, 17(12), 1425-1438. 156856206778937208

Gourmelon, G. (2015). Global Plastic Production Rises, Recycling Lags. Retrieved May 6, 2016, from http:// vitalsigns.worldwatch.org/vs-trend/global-plasticproduction-rises-recycling-lags

Grousseau, E., Blanchet, E., Déléris, S., Albuquerque, M. G. E., Paul, E., \& Uribelarrea, J. L. (2014). Phosphorus limitation strategy to increase propionic acid flux towards 3-hydroxyvaleric acid monomers in Cupriavidus necator. Bioresource Technology, 153, 206-215. j.biortech.2013.11.072

Guell, A. F. (2015). First year report: Polyhydroxyalkanoate production with model organism Cupriavidus necator and extreme halophile Haloferax mediterranei. The University of Manchester, Manchester, UK.

Han, J., Wu, L. P., Hou, J., Zhao, D., \& Xiang, H. (2015). Biosynthesis, characterization, and hemostasis potential of tailor-made poly(3-hydroxybutyrate- co 3-hydroxyvalerate) produced by Haloferax mediterranei. Biomacromolecules, 16(2), 578-588. https://doi.org/10.1021/bm5016267

Hermann-Krauss, C., Koller, M., Muhr, A., Fasl, H., Stelzer, F., \& Braunegg, G. (2013). Archaeal production of polyhydroxyalkanoate (PHA) Co- and terpolyesters from biodiesel industry-derived by-products. Archaea, 2013. https://doi.org/10.1155/2013/129268

Kaewkannetra, P. (2012). Fermentation of sweet sorghum into added value biopolymer of polyhydroxyalkanoates (PHAs). In C. J. R. Verbeek (Ed.), Products and Applications of Biopolymers (pp. 41-60). Rijeka, Croatia: InTech. https://doi.org/ $10.5772 / 1802$

Koller, M., Chiellini, E., \& Braunegg, G. (2015). Study on the production and Re-use of Poly(3-hydroxybutyrateco-3- hydroxyvalerate) and extracellular polysaccharide by the Archaeon Haloferax mediterranei strain DSM 1411. Chemical and Biochemical Engineering Quarterly, 29(2), 87-98. https://doi.org/10.15255/CABEQ.2014.2058
Koller, M., Hesse, P., Bona, R., Kutschera, C., Atliæ, A., \& Braunegg, G. (2007). Biosynthesis of high quality polyhydroxyalkanoate Co- And terpolyesters for potential medical application by the archaeon Haloferax mediterranei. Macromolecular Symposia, 253, 33-39. https://doi.org/10.1002/masy.200750704

Koller, M., Salerno, A., Muhr, A., Reiterer, A., \& Braunegg, G. (2012). Polyhydroxyalkanoates: Biodgradable polymers and plastics from renewable resources. Materials and Technology, 46(6), 23-30.

Lillo, J. G., \& Rodriguez-Valera, F. (1990). Effects of Culture Conditions on Poly(beta-Hydroxybutyric Acid) Production by Haloferax mediterranei. Applied and Environmental Microbiology, 56(8), 2517-2521.

Pal, K., Paulson, A. T., \& Rousseau, D. (2009). Biopolymers in controlled-release delivery systems. In Handbook of Biopolymers and Biodegradable Plastics (pp. 519-557). https://doi.org/10.1016/B9780-12-374195-0.00016-1

Rodriguez-Valera, F., Ruiz-Berraquero, F., \& RamosCormenzana. (1980). Short Communication. Isolation of extremely halophilic bacteria able to grow in defined inorganic media with single carbon sources. Microbiology, 119(2), 535-538. https:// doi.org/10.1099/00221287-119-2-535

Rudnik, E. (2013). Compostable Polymer Materials: definitions, structures, and methods of preparation. In Handbook of Biopolymers and Biodegradable Plastics (pp. 189-211). Elsevier. https://doi.org/ 10.1016/B978-1-4557-2834-3.00010-0

Sankhla, I. S., Bhati, R., Singh, A. K., \& Mallick, N. (2010). Poly(3-hydroxybutyrate-co-3-hydroxyvalerate) copolymer production from a local isolate, Brevibacillus invocatus MTCC 9039. Bioresource Technology, 101(6), 1947-1953. https://doi.org/10.1016/ j.biortech.2009.10.006

Saharan, B.S., Grewal, A. \& Kumar, P. (2014). Biotechnological production of polyhydroxy alkanoates: A Review on trends and latest developments. Chinese Journal of Biology, 2014, 118. https://doi.org/10.1155/2014/802984

Wang, H. J., Zhou, W. H., Yin, X. F., Zhuang, Z. X., Yang, H. H., \& Wang, X. R. (2006). Template synthesized molecularly imprinted polymer nanotube membranes for chemical separations. Journal of the American Chemical Society, 128(50), 15954-15955. https://doi.org/10.1021/ja065116v

Wang, Q., Liu, X., \& Qi, Q. (2014). Biosynthesis of poly(3hydroxybutyrate-co-3-hydroxyvalerate) from glucose with elevated 3-hydroxyvalerate fraction via combined citramalate and threonine pathway in Escherichia coli. Applied Microbiology and Biotechnology, 98(9), 3923-3931. https://doi.org/10.1007/s00253-0135494-5 